\title{
Killer Immunoglobulin-Like Receptor 2DS2 (KIR2DS2), KIR2DL2-HLA-C1, and KIR2DL3 as Genetic Markers for Stratifying the Risk of Cytomegalovirus Infection in Kidney Transplant Recipients
}

\author{
Dominika Deborska-Materkowska ${ }^{1}$, Agnieszka Perkowska-Ptasinska ${ }^{1}$, \\ Anna Sadowska-Jakubowicz ${ }^{1}$, Jolanta Gozdowska ${ }^{1}$, Michał Ciszek ${ }^{2}$, Joanna Pazik ${ }^{1}$ (1), \\ Agata Ostaszewska ${ }^{3}$, Maciej Kosieradzki ${ }^{3}$, Jacek Nowak ${ }^{4}$ (D) and Magdalena Durlik ${ }^{1, *}$ \\ 1 Department of Transplantation Medicine, Nephrology, Internal Diseases, T. Orłowski Institute of \\ Transplantation Medical University of Warsaw, 59 Nowogrodzka Street, 02-006 Warsaw, Poland; \\ dominika.deborska-materkowska@wum.edu.pl (D.D.-M.); \\ agnieszka.perkowska-ptasinska@wum.edu.pl (A.P.-P.); andziaa@wp.pl (A.S.-J.); jola-md@prokonto.pl (J.G.); \\ jpazik@poczta.fm (J.P.) \\ 2 Department of Immunology, Transplantology, Internal Diseases, T. Orłowski Institute of Transplantation \\ Medical University of Warsaw, 59 Nowogrodzka Street, 02-006 Warsaw, Poland; mciszek@onet.pl \\ 3 Department of General and Transplant Surgery, T. Orłowski Institute of Transplantation Medical University \\ of Warsaw, 59 Nowogrodzka Street, 02-006 Warsaw, Poland; ag.ostaszewska@gmail.com (A.O.); \\ mpkosieradzki@gmail.com (M.K.) \\ 4 Department of Immunogenetics Institute of Hematology and Transfusion Medicine 14 Indira Gandhi Street, \\ 02-776 Warsaw, Poland; jnowak@ihit.waw.pl \\ * Correspondence: magdalena.durlik@wum.edu.pl
}

Received: 18 December 2018; Accepted: 24 January 2019; Published: 28 January 2019

\begin{abstract}
Infection with cytomegalovirus (CMV) remains a major problem in kidney transplant recipients, resulting in serious infectious complications and occasionally mortality. Accumulating evidence indicates that natural killer cell immunoglobulin-like receptors (KIRs) and their ligands affect the susceptibility to various diseases, including viral infections (e.g., CMV infection). We investigated whether KIR genes and their ligands affect the occurrence of CMV infection in a group of 138 kidney transplant recipients who were observed for 720 days posttransplantation. We typed the recipients for the presence of KIR genes (human leukocyte antigen C1 [HLA-C1], HLA-C2, HLA-A, HLA-B, and HLA-DR1) by polymerase chain reaction with sequence-specific primers. The multivariate analysis revealed that the lack of KIR2DS2 $(p=0.035)$, the presence of KIR2DL3 $(p=0.075)$, and the presence of KIR2DL2-HLA-C1 $(p=0.044)$ were risk factors for posttransplant CMV infection. We also found that a lower estimated glomerular filtration rate $(p=0.036)$, an earlier time of antiviral prophylaxis initiation $(p=0.025)$, lymphocytopenia $(p=0.012)$, and pretransplant serostatus (donor-positive/recipient-negative; $p=0.042$ ) were independent risk factors for posttransplant CMV infection. In conclusion, our findings confirm that the KIR/HLA genotype plays a significant role in anti-CMV immunity and suggest the contribution of both environmental and genetic factors to the incidence of CMV infection after kidney transplantation.
\end{abstract}

Keywords: killer-cell immunoglobulin-like receptor; cytomegalovirus; natural killer cell; human leukocyte antigen; lymphocytopenia; kidney transplantation 


\section{Introduction}

Despite the introduction of novel diagnostic and therapeutic modalities for the management of cytomegalovirus (CMV), it remains a significant cause of serious posttransplant complications, with high rates of mortality and graft loss [1]. Donor (D) and recipient (R) CMV serostatus is an important determinant of the risk of CMV infection posttransplantation. Although serostatus is useful, it does not allow us to precisely predict who is at risk for infection. Despite the implementation of prevention strategies that are based on pretransplant $\mathrm{D} / \mathrm{R}$ serostatus, a high incidence of late-onset CMV has occurred in all studies that have evaluated universal prophylaxis. Apart from pretransplant serology, other risk factors for CMV infection include certain types of transplant (e.g., lung), shorter courses of prophylaxis, disease severity, higher levels of immunosuppression, transplant failure, allograft rejection, and the presence of immune reconstitution [2-5].

The immune response to CMV infection is complex. The role of both adaptive and innate responses has not been well established in transplant recipients. Natural killer (NK) cells are effector cells that constitute a crucial part of the innate immune system and provide the first-line of defense against and control of CMV infection [6]. The inhibition and activation of the NK cell response is modulated by signals that include the interplay between polymorphic killer-cell immunoglobulin-like receptors (KIRs) and their ligands, human leukocyte antigen (HLA) class I molecules. There are two types of KIR molecules (activating and inhibitory) that are expressed on NK cells and minor subpopulations of $\mathrm{T}$ cells. KIRs that have long cytoplasmic tails (killer-cell immunoglobulin-like receptor-2DL1 (KIR2DL1), KIR2DL2, KIR2DL3, KIR2DL5, KIR3DL1, KIR3DL2, and KIR3DL3) suppress effector functions. KIRs that have short intracytoplasmic tails (KIR2DS1, KIR2DS2, KIR2DS3, KIR2DS4, KIR2DS5, and KIR3DS1) activate lymphocytes through an associated DAP12 molecule [7-9]. KIR2DL4 encodes a receptor that performs both inhibitory and activating functions. Inhibitory KIRs recognize HLA class I molecules on the surface of target cells. Activating KIRs have lower affinity for HLA, and their natural ligands are less well documented. There are two categories of KIR haplotypes. Group A haplotypes carry mostly inhibitory KIRs; only KIR2DS4 and KIR2DL4 are activating ones. B-type haplotypes are more variable and contain more than one activating KIR gene other than KIR2DS4 [10]. KIR and HLA genes have been identified on two separate chromosomes (chromosomes 19 and 6, respectively) and, thus, are inherited independently. Therefore, an individual may lack the corresponding HLA ligands for KIRs. Depending on KIR and KIR ligand genotypes, people may differ substantially in their NK response. The lack of ligands for inhibitory KIRs and the presence of activating KIRs in the recipient have been associated with a protective effect on the rate of CMV infection after kidney transplantation [11,12].

The aim of the present study was to analyze the association between post-kidney transplant CMV infection and the recipient's KIR genotype and evaluate other possible risk factors for the occurrence of CMV infection in this patient population.

\section{Results}

\subsection{Clinical Characteristics of the Study Patients}

One hundred twenty-eight study participants received an organ from a deceased donor, and eight participants received an organ from a living related donor. Cytomegalovirus infection occurred in $36.23 \%$ of the 138 patients during 720 days after kidney transplantation. The pretransplant $D / R$ serostatus and baseline recipients' characteristics are summarized in Table 1. 
Table 1. Baseline characteristics of the study patients.

\begin{tabular}{|c|c|}
\hline Characteristic & \\
\hline Age of recipient (years) (median (range)) & $48(20-77)$ \\
\hline Gender (male) & $n=90(65 \%)$ \\
\hline \multicolumn{2}{|l|}{ Type of Transplant } \\
\hline Kidney & $n=133(96.4 \%)$ \\
\hline Kidney + pancreas & $n=4(2.9 \%)$ \\
\hline Kidney + heart & $n=1(0.7 \%)$ \\
\hline \multicolumn{2}{|l|}{ Type of Donor } \\
\hline Living & $n=10(7.2 \%)$ \\
\hline Deceased & $n=128(92.8 \%)$ \\
\hline \multicolumn{2}{|l|}{ Pretransplant Donor (D)/Recipient (R) CMV Serostatus } \\
\hline $\mathrm{D}+/ \mathrm{R}-$ & $n=34(25.2 \%)$ \\
\hline $\mathrm{D}-$ or $\mathrm{D}+/ \mathrm{R}+$ & $n=104(74.8 \%)$ \\
\hline \multicolumn{2}{|l|}{ Induction Therapy } \\
\hline Thymoglobulin & $n=13(9.4 \%)$ \\
\hline Basiliximab & $n=72(52.2 \%)$ \\
\hline None & $n=53(38.4 \%)$ \\
\hline \multicolumn{2}{|l|}{ Maintenance Immunosuppression } \\
\hline tacrolimus + mycophenolate mofetil/sodium + prednisone & $n=121(87.7 \%)$ \\
\hline cyclosporine A + mycophenolate mofetil/sodium + prednisone & $n=15(10.9 \%)$ \\
\hline tacrolimus + everolimus + prednisone & $n=2(1.4 \%)$ \\
\hline Time from kidney transplant to antiviral prophylaxis initiation (days) (mean $\pm \mathrm{SD}$ [range]) & $6 \pm 5(0-25)$ \\
\hline Time from kidney transplant to antiviral prophylaxis discontinuation (days) (mean \pm SD [range]) & $90 \pm 21(12-178)$ \\
\hline Duration of antiviral prophylaxis (days) (mean \pm SD (range)) & $84 \pm 21(10-175)$ \\
\hline \multicolumn{2}{|l|}{ Allograft function $\left(\mathrm{eGFR} ; \mathrm{ml} / \mathrm{min} / 1.73 \mathrm{~m}^{2}\right)($ mean $\pm \mathrm{SD}$ [range] $)$} \\
\hline Day 30 & $46.7 \pm 19.9(6.7-103.7)$ \\
\hline Day 90 & $47.8 \pm 18.5(8.5-98.9)$ \\
\hline Day 360 & $49.5 \pm 18.6(8.8-105.0)$ \\
\hline \multicolumn{2}{|l|}{ KIR Genotype } \\
\hline $\mathrm{A} / \mathrm{A}$ & $n=42(30.4 \%)$ \\
\hline $\mathrm{B} / \mathrm{X}$ & $n=96(69.6 \%)$ \\
\hline
\end{tabular}

eGFR, estimated glomerular filtration rate; SD, standard deviation.

KIR2DL4, KIR3DP1, KIR3DL2, and KIR3DL3 were present in all of the recipients. The frequencies of other KIR genes are presented in Table 2.

Table 2. Frequency of each KIR gene in 138 patients.

\begin{tabular}{cccccccccccccc}
\hline & \multicolumn{10}{c}{ KIR Genes } \\
\cline { 2 - 14 } & 3DS1 & 2DL1 & 2DL2 & 2DL3 & 2DL5 & 2DS1 & 2DS2 & 2DS3 & 2DS4fl & 2DS4del & 2DS5 & 3DL1 & 2DP1 \\
\hline$N$ & 48 & 130 & 71 & 115 & 64 & 51 & 72 & 42 & 63 & 106 & 37 & 128 & 132 \\
\hline$\%$ & 34.8 & 94.2 & 51.4 & 83.3 & 46.4 & 36.9 & 52.2 & 30.4 & 45.7 & 76.8 & 26.8 & 92.8 & 95.7 \\
\hline
\end{tabular}

2.2. The Relation between the Cmv Infection Occurrence and Individual Kir Gene Frequencies (Univariate Analysis)

We analyzed whether individual KIR genes influence the incidence of CMV infection. The KIR gene distribution between the CMV+ and CMV - groups is presented in Figure 1. 


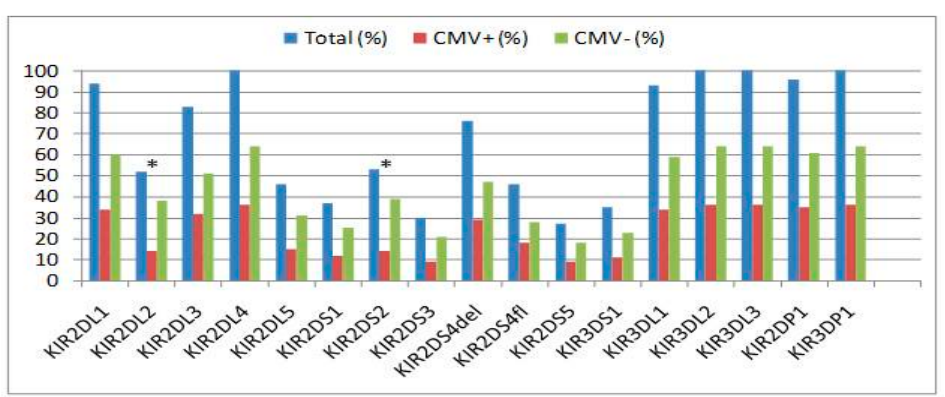

Figure 1. Frequency of $\mathrm{CMV}$ infection $(\mathrm{CMV}+)$ relative to each killer cell immunoglobulin-like receptor gene. The asterisk $\left(^{*}\right)$ indicates statistical significance.

The KIR2DS2 gene frequency was $73.6 \%$ in the CMV - group vs. $26.4 \%$ in the CMV+ group $(p=0.012)$. The KIR2DL2 gene frequency was $73.2 \%$ in the CMV- group vs. $26.8 \%$ in the CMV+ group $(p=0.017)$. We found nearly absolute concordance between KIR2DS2 and KIR2DL2 with regard to their presence. Therefore, these two genes, with the exception of one individual, had identical frequencies. No significant difference was found in the other KIR gene frequencies between the CMV+ and CMVgroups (Figure 1).

\subsection{KIR Genotype and Rate of Cmv Infection (Univariate Analysis)}

Based on the presence or absence of multiple activating KIRs, we categorized the genotypes as AA (containing KIR2DS4 as the only activating gene) or B/X type (carrying activating KIR genes other than KIR2DS4), which is in accordance with the published literature [10,13]. Forty-two $(30.4 \%)$ individuals were assigned to the KIR A/A genotype; the remaining 96 (69.6\%) individuals were assigned to the KIR B/X genotype. We noted a trend toward a lower incidence of CMV infection in recipients who carried the KIR B/X genotype $(n=30[31.2 \%])$ compared with the KIR A/A genotype $(n=20[47.6 \%])$. Nevertheless, the difference between both KIR genotypes did not reach statistical significance $(p=0.065$; Table 3$)$.

Table 3. Association between KIRs and KIR genotypes and CMV infection.

\begin{tabular}{|c|c|c|c|}
\hline Genetic factor & CMV Infection $n(\%)$ & No CMV Infection $n(\%)$ & $p$ \\
\hline \multicolumn{3}{|c|}{ KIR Genotype } & \multirow{3}{*}{0.065} \\
\hline $\mathrm{A} / \mathrm{A}$ & $20(47.6)$ & $22(52.4)$ & \\
\hline $\mathrm{B} / \mathrm{X}$ & $30(31.2)$ & $66(68.8)$ & \\
\hline \multicolumn{3}{|c|}{ Number of Activating KIRs } & \multirow{7}{*}{0.201} \\
\hline 1 & $20(47.6)$ & $22(52.4)$ & \\
\hline 2 & $8(25.8)$ & $23(74.2)$ & \\
\hline 3 & $7(36.8)$ & $12(63.2)$ & \\
\hline 4 & $10(45.5)$ & $12(54.5)$ & \\
\hline 5 & $4(23.5)$ & $13(76.5)$ & \\
\hline 6 & $1(14.3)$ & $6(85.7)$ & \\
\hline \multicolumn{3}{|c|}{ Number of Inhibitory KIRs } & \multirow{5}{*}{0.631} \\
\hline 5 & $2(40.0)$ & $3(60.0)$ & \\
\hline 6 & $23(42.6)$ & $31(57.4)$ & \\
\hline 7 & $19(32.2)$ & $40(67.8)$ & \\
\hline 8 & $6(30.0)$ & $14(70.0)$ & \\
\hline KIR2DL1-HLA-C2 & $29(37.2)$ & $49(62.8)$ & 0.791 \\
\hline KIR2DL2-HLA-C1 & $17(30.9)$ & $38(69.1)$ & 0.289 \\
\hline KIR2DL3-HLA-C1 & $34(40.0)$ & $51(60.0)$ & 0.243 \\
\hline KIR3DL1-HLA-Bw4 & $35(37.2)$ & $59(62.8)$ & 0.720 \\
\hline KIR3DL2-HLA-A3/A11 & $22(38.6)$ & $35(61.4)$ & 0.627 \\
\hline
\end{tabular}

KIR, killer-cell immunoglobulin-like receptor; HLA, human leukocyte antigen. 
2.4. Cumulative Number of Activating and Inhibitory Genes That Were Present in Kir Haplotypes and Cmv Infection (Univariate Analysis)

We analyzed whether the cumulative number of either activating or inhibitory KIRs in the recipient's genome varied between $\mathrm{CMV}+$ and $\mathrm{CMV}-$ patients. We did not find such a difference (Figure 2a,b).

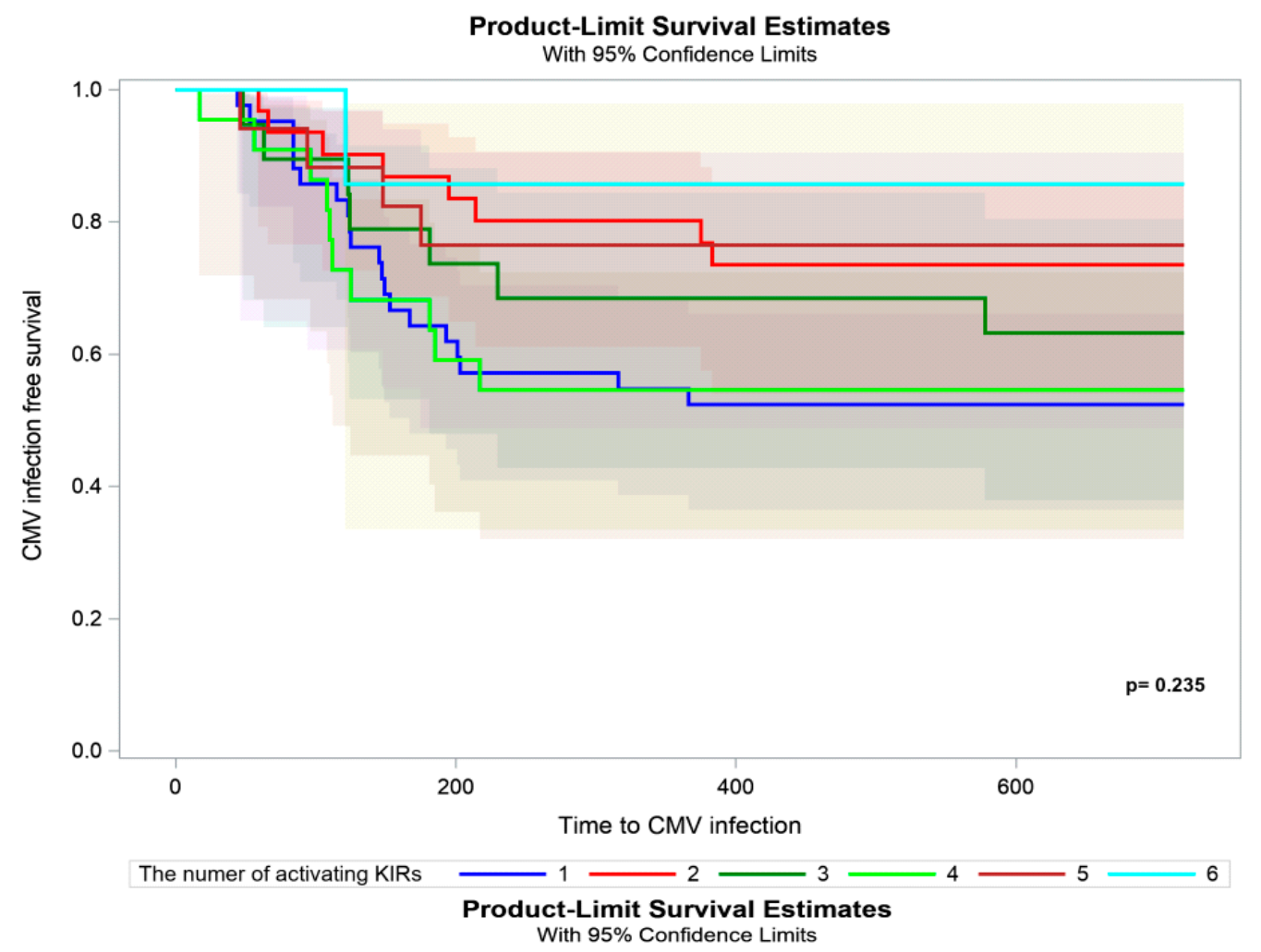

(a)

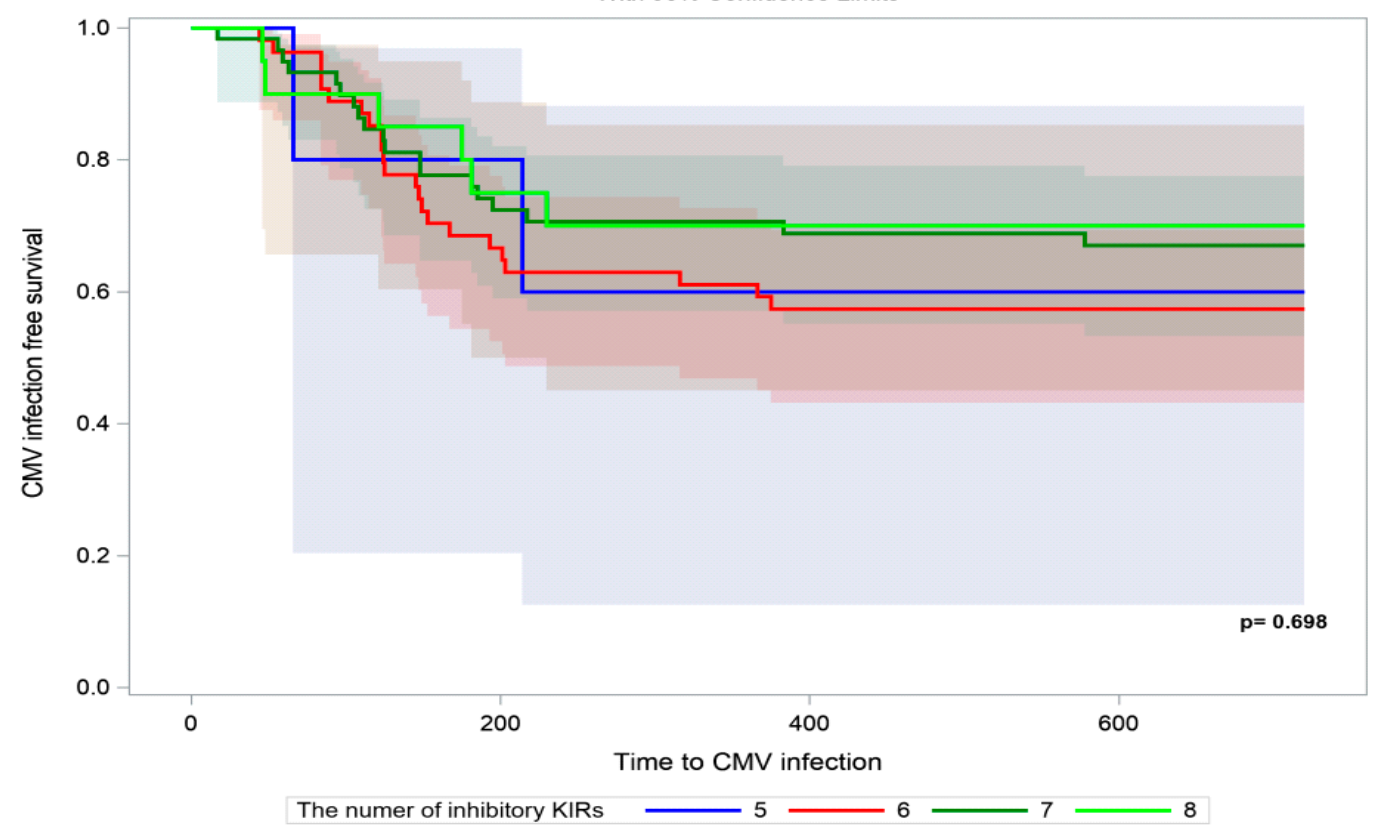

(b)

Figure 2. Occurrence of CMV infection in kidney transplant recipients stratified by the cumulative number of (a) activating and (b) inhibitory KIR genes. 


\subsection{KIR/HLA Interactions and CMV Infection (Univariate Analysis)}

To determine whether certain receptor-ligand combinations influence the development of CMV infection, we calculated the number of matches for certain KIRs with known HLA ligands. Univariate analysis did not reveal significant associations between certain KIR/HLA matches and posttransplant CMV infection (Table 3).

\subsection{Factors Other Than KIR Factors That Affect Posttransplant Cmv Infection}

Univariate logistic regression analysis was performed to examine factors that could potentially impact the posttransplant occurrence of CMV infection. Of these factors, allograft function $(p=0.04$ for recipients with a lower GFR on day 30; $p=0.0006$ for recipients with a lower GFR on day 90), lymphocytopenia before day 90 posttransplantation $(p=0.0003)$, leucopenia $(p=0.007)$, higher pretransplant recipient CMV IgG titer $(p=0.016)$, pretransplant D/R CMV serostatus $(p=0.019$ for $\mathrm{D}+/ \mathrm{R}-)$, and an earlier time of antiviral prophylaxis initiation $(p=0.005)$ were statistically significant. All of other variables were not associated with CMV infection (Table 4).

Table 4. Clinical characteristics of kidney transplant recipients stratified by the occurrence of CMV infection.

\begin{tabular}{|c|c|c|c|}
\hline Characteristic & CMV Infection & No CMV Infection & $p$ \\
\hline Age (years) (mean $\pm \mathrm{SD})$ & $50.3 \pm 13.6$ & $46.5 \pm 14.6$ & 0.143 \\
\hline Gender, male (n [\%]) & $30(60.0)$ & $60(68.2)$ & 0.332 \\
\hline \multicolumn{4}{|c|}{ Type of Transplant (n [\%]) } \\
\hline Kidney & $48(36.1)$ & $85(63.9)$ & \multirow{3}{*}{0.371} \\
\hline Kidney + pancreas & $1(25)$ & $3(75)$ & \\
\hline Kidney + heart & $1(100)$ & 0 & \\
\hline Donor (deceased/living) (n [\%]) & $49(38.3) / 1(10)$ & $79(61.7) / 9(90)$ & 0.073 \\
\hline \multicolumn{4}{|c|}{ Allograft Function (eGFR) (mean \pm SD) } \\
\hline Day 30 & $41.6 \pm 16.4$ & $49.6 \pm 21.1$ & 0.040 \\
\hline Day 90 & $40.9 \pm 17.1$ & $51.6 \pm 18.2$ & 0.0006 \\
\hline \multicolumn{4}{|c|}{ Acute Rejection (n [\%]) } \\
\hline TCMR & $18(42.9)$ & $24(57.1)$ & 0.639 \\
\hline ABMR & $8(47.1)$ & $9(52.9)$ & 0.521 \\
\hline TCMR or ABMR & $22(44.9)$ & $27(55.1)$ & 0.362 \\
\hline \multicolumn{4}{|c|}{ HLA Mismatch (n [\%]) } \\
\hline$\leq 3 / 6$ & $25(40.3)$ & $37(59.7)$ & \multirow{2}{*}{0.366} \\
\hline$>3 / 6$ & $25(32.9)$ & $51(67.1)$ & \\
\hline \multicolumn{4}{|c|}{ Lymphocyte blood count $\leq$ day 90 (G/L) (n [\%]) } \\
\hline$<0.8$ & $31(56.4)$ & $24(43.6)$ & \multirow{2}{*}{0.0003} \\
\hline$\geq 0.8$ & $17(24.6)$ & $52(75.4)$ & \\
\hline \multicolumn{4}{|c|}{ Leukocyte Blood Count $\leq$ Day 90 (G/L) (n [\%]) } \\
\hline$<4.1$ & $34(46.6)$ & $39(53.4)$ & \multirow{2}{*}{0.007} \\
\hline$\geq 4.1$ & $16(24.6)$ & $49(75.4)$ & \\
\hline \multicolumn{4}{|c|}{ Donor (n [\%]) } \\
\hline CMV IgG+ & $45(37.5)$ & $75(62.5)$ & \multirow{2}{*}{0.752} \\
\hline CMV IgG- & $5(33.3)$ & $10(66.7)$ & \\
\hline \multicolumn{4}{|c|}{ Pretransplant Recipient CMV Serostatus (n [\%]) } \\
\hline CMV IgG+ & $32(31.7)$ & $69(68.3)$ & \multirow{2}{*}{0.066} \\
\hline CMV IgG- & $18(48.7)$ & $19(51.3)$ & \\
\hline
\end{tabular}


Table 4. Cont

\begin{tabular}{|c|c|c|c|}
\hline Characteristic & CMV Infection & No CMV Infection & $p$ \\
\hline $\begin{array}{l}\text { Pretransplant recipient CMV IgG titer day } 0(\mathrm{AU} / \mathrm{mL}) \\
(\text { mean } \pm \mathrm{SD})\end{array}$ & $80.7(95.9)$ & $133.4(116.6)$ & 0.016 \\
\hline \multicolumn{4}{|c|}{ Pretransplant Donor/Recipient CMV Serostatus (n [\%]) } \\
\hline $\mathrm{D}+/ \mathrm{R}-$ & $18(52.9)$ & $16(47.1)$ & \multirow{2}{*}{0.019} \\
\hline $\mathrm{D}+$ or $\mathrm{D}-/ \mathrm{R}+$ & $32(30.8)$ & $72(69.2)$ & \\
\hline Duration of antiviral prophylaxis (days) (mean \pm SD) & $80 \pm 23$ & $87 \pm 19$ & 0.276 \\
\hline $\begin{array}{l}\text { Time from kidney transplant to antiviral prophylaxis } \\
\text { initiation (days) (mean } \pm \text { SD) }\end{array}$ & $4.68 \pm 4.97$ & $7.22 \pm 5.39$ & 0.005 \\
\hline \multicolumn{4}{|c|}{ Induction Therapy (\%) } \\
\hline Yes (basiliximab) & 27.8 & 72.2 & \multirow{3}{*}{0.076} \\
\hline Yes (thymoglobulin) & 53.8 & 46.2 & \\
\hline No & 43.4 & 56.6 & \\
\hline \multicolumn{4}{|c|}{ Induction Therapy (\%) } \\
\hline Yes (basiliximab or thymoglobulin) & 31.8 & 68.2 & \multirow{2}{*}{0.166} \\
\hline No & 43.4 & 56.6 & \\
\hline
\end{tabular}

eGFR, estimated glomerular filtration rate; TCMR, T-cell mediated rejection; ABMR, antibody-mediated rejection; HLA, human leukocyte antigen; SD, standard deviation; AU, arbitrary units.

\subsection{Independent Risk Factors for Posttransplant Cmv Infection (Multivariate Analysis)}

The multivariate analysis included all clinical variables and individual KIR genes and their HLA ligands that were analyzed in the univariate analyses. The maximum model to be considered was identified. The optimal subset of variables was then selected, and their reliability was assessed. KIR2DL2 and KIR2DS2 are in strong linkage disequilibrium and are expected to be expressed together. In the present study KIR2DL2 and KIR2DS2 were present in the same group of individuals, with the exception of one recipient. Therefore, statistical models could include either KIR2DL2 or KIR2DS2. The multivariate analysis revealed the following independent risk factors for posttransplant CMV infection: lack of KIR2DS2, presence of KIR2DL2-HLA-C1 and KIR2DL3, allograft dysfunction, earlier time of antiviral prophylaxis initiation, lymphocytopenia before day 90 posttransplantation, and high-risk D/R serostatus (Table 5).

Table 5. Best subset of variables with the strongest predictive value for the incidence of posttransplant $\mathrm{CMV}$ infection according to the multivariate analysis.

\begin{tabular}{cccc}
\hline \multirow{2}{*}{ Best Subset of Parameters } & \multicolumn{3}{c}{ Multivariate Analysis } \\
\cline { 2 - 4 } & OR & 95\% CI & $p$ \\
\hline Lack of KIR2DS2 & 7.984 & $1.155-55.215$ & 0.04 \\
\hline KIR2DL2-HLA-C1 & 8.197 & $1.055-62.500$ & 0.04 \\
\hline KIR2DL3 & 4.219 & $0.866-20.833$ & 0.08 \\
\hline Allograft dysfunction & 1.030 & $1.002-1.059$ & 0.04 \\
\hline Lymphocytopenia & 3.200 & $1.286-7.960$ & 0.01 \\
\hline Earlier time of antiviral prophylaxis initiation & 1.107 & $1.013-1.212$ & 0.03 \\
\hline D+/R- pretransplant serostatus & 3.241 & $1.043-10.069$ & 0.04 \\
\hline
\end{tabular}

KIR, killer-cell immunoglobulin-like receptor; CI, confidence interval; OR, odds ratio; D, donor; R, recipient.

A Kaplan Meier curve of the time to CMV infection in recipients with KIR2DS2 vs. recipients without KIR2DS2 is shown in Figure 3. 


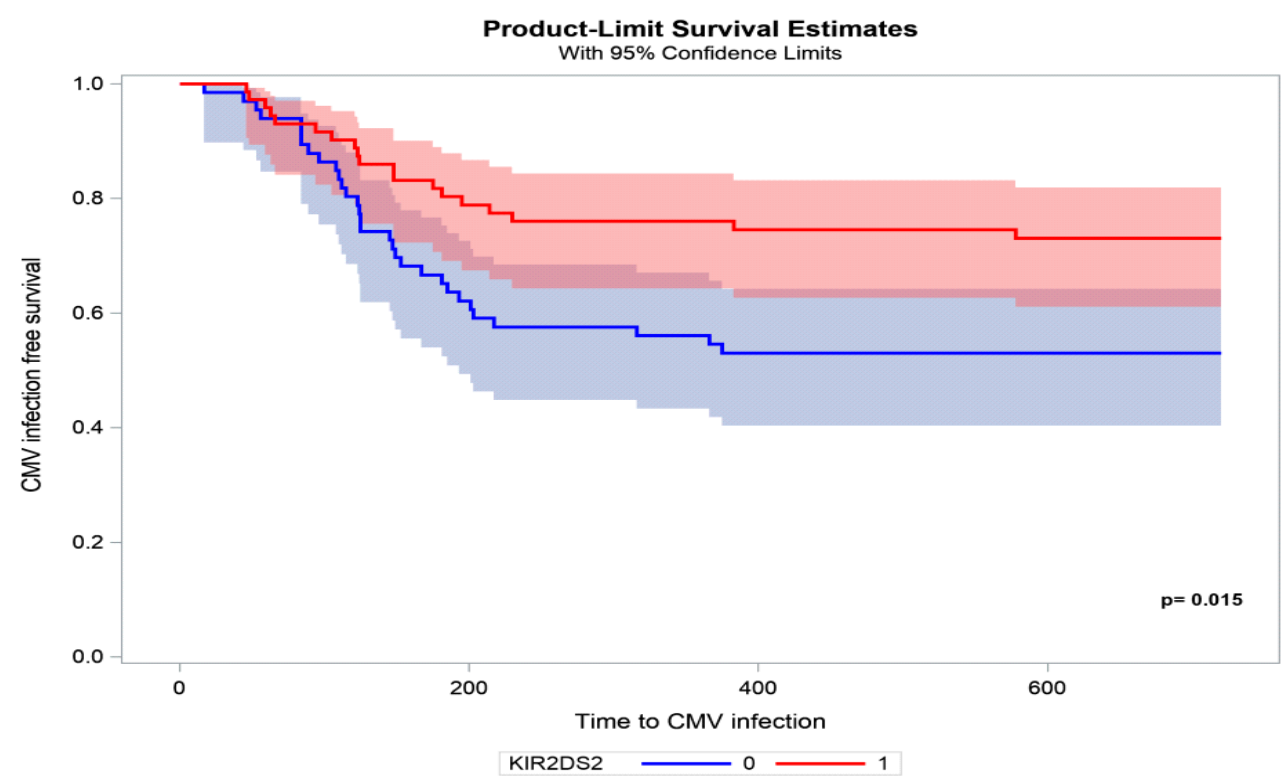

Figure 3. Time from kidney transplantation to CMV infection in patients with (red line) vs. without (blue line) KIR2DS2.

\section{Discussion}

The present study analyzed the association between KIR genotype diversity in kidney transplant recipients and the occurrence of CMV infection. The key finding of this study was the association between the lack of activating KIR2DS2 and posttransplant CMV infection. This phenomenon was also described in patients after hematopoietic cell transplantation (HCT). In the study by Zaia et al., the presence of KIR2DS2 and KIR2DS4 in the donor genotype was associated with lower CMV infection in HCT recipients [14]. This finding was supported by the fact that upregulation of the KIR expression of 2DS2 and 2DS4 protected against CMV reactivation in the early post-HCT time. KIR2DS2 and KIR2DS4 expression was elevated in individuals after HCT compared with donor expression prior to transplant and in recipients with CMV DNAemia compared with non-viremic recipients [15]. In another study of a D+/R - organ transplant recipient cohort, the presence of KIR2DS2 in combination with KIR2DL3 was associated with protection against CMV viremia. However, this protective effect was only present if neither the donor nor the recipient expressed any HLA-C2 molecules [16]. In immunocompetent individuals, KIR2DS2 was found to be negatively associated with the risk of infection, in which it was more frequent in controls than in those with CMV infection $(\mathrm{OR}=0.14)$ [17]. Additionally, the binding of KIR2DS2 to different peptides that derive from diverse pathogens and to different HLA class I molecules is particularly interesting given its protective role in acute viral encephalitis and chronic hepatitis $C$ virus infection [18-20]. Interactions between viral epitopes, KIRs that are expressed on NK cells, and HLA ligands can lead to either the activation or inhibition of cytotoxic NK cell activity. KIR and HLA genotypes segregate independently of each other. Therefore, a proportion of KIRs likely has no HLA ligand. Conversely, individuals may possess HLA ligands for which they have no KIR. Group 1 HLA-C (HLA-C1) allotypes are characterized by an asparagine residue at position 80 . They are ligands for the inhibitory receptors KIR2DL2 and KIR2DL3, which segregate as alleles of a single locus. KIR2DL2 binds HLA-C1 with greater affinity than KIR2DL3 [21]. Our results showed that subjects who had KIR2DL3 and KIR2DL2-HLA-C1 were more likely to develop CMV infection. These findings are consistent with previous reports of KIR and HLA genotyping, indicating that the absence of HLA ligands for inhibitory KIRs could be associated with a significant reduction of CMV infection [11]. The cumulative evidence shows that KIR2DL2-HLA-C1 and KIR2DL3 may be molecular biomarkers of virus-induced diseases [22-24].

To date, it has been difficult to demonstrate the binding of KIR2DS2 to HLA-C1. The sequences of KIR2DS2 are very similar to KIR2DL2. Despite shared sequence homology, one key difference between 
KIR2DS2 and KIR2DL2 is the presence of a tyrosine (compared with a phenylalanine) residue at position 45, and this is thought to substantially affect the binding of KIR2DS2 to HLA-C [25]. Steward et al. examined the binding of activating KIR to cells that were infected with human herpes viruses, including CMV, inducing no detectable KIR2DS2 ligand at the cell surface [26]. A very low affinity of the KIR2DS2-HLA-C interaction that does not have the same degree of peptide selectivity as other KIR-HLA interactions was the reason that we did not analyze KIR2DS2-HLA-C. We are aware that the lack of information concerning KIR2DS2 ligands may be a limitation of the present study.

Other authors did not find that KIR and HLA genotypes are related to protection against CMV infection in kidney transplant recipients [27]. One possible explanation for this finding is that the previous study analyzed a patient cohort with a high risk of CMV infection (i.e., exclusively CMV-negative recipients of a CMV-positive donor kidney). KIR2DL2 and KIR2DS2 belong to the KIR $\mathrm{B}$ haplotype. The majority $(74.8 \%)$ of our recipients were CMV-seropositive before transplantation. Evidence indicates that KIR B haplotypes modify the risk of CMV viremia solely in patients who have already been exposed to CMV before organ transplantation, suggesting that "primed" or "memory-like" NK cells are cellular correlates for this protective effect [28].

The incidence of CMV infection or reactivation was proposed to be increased by the heritance of multiple activating KIR genes [12,29]. We observed a trend toward a lower incidence of CMV infection in recipients who carried the KIR B/X genotype, but no significant difference was found between both the KIR A/A and KIR B/X genotypes $(p=0.065)$. The degree of protection against CMV was also suggested to increase with the number of activating KIR genes [12]. We analyzed the potential additive protective effect of the number of activating KIRs relative to CMV occurrence, but we did not confirm such an association.

In the present study, a protective effect against CMV infection in recipients who had the KIR2DS2 genotype was found in $73.6 \%$ of the individuals, but $26.4 \%$ who had this protective genotype still had CMV infection. This may be attributable to the fact that the KIR genotype profile does not necessarily correlate with concurrent expression. The expression of KIR2DS2 was previously shown to vary among genotype-positive healthy individuals, in which some were expressers and some were non-expressers [15]. We did not investigate the expression of KIRs, which may be considered a limitation of our study.

Similar to previous reports, the present study confirmed that donor and recipient (D/R) CMV serostatus and allograft dysfunction were key predictors of the risk of CMV infection after kidney transplantation $[2,3,5,30]$. Renal insufficiency is associated with immune dysfunction, including lymphocytopenia, which contributes to the high prevalence of infections [31]. The present study confirmed our previous findings that total lymphocyte count before posttransplant day 90 is a risk factor for posttransplant CMV infection [32]. This phenomenon has also been described in liver transplant patients [33,34].

Interestingly, in the present study, an earlier time of antiviral prophylaxis initiation was not significant in the univariate analysis, but it became a significant predictor of posttransplant CMV infection in the multivariate analysis. Antiviral prophylaxis that is initiated promptly after transplantation could impair the development of an adequate CMV-specific response of cytotoxic and helper T lymphocytes, which seems to be crucial for protection against CMV infection. This finding is consistent with another study that found that a 14-day delay in the initiation of long-term prophylaxis in $(\mathrm{D}+/ \mathrm{R}-)$ organ transplant recipients could prevent the development of late CMV infection [35].

\section{Materials and Methods}

\subsection{Study Design, Participants, and Outcome Parameters}

Patients who received a kidney transplant between March 2007 and November 2014 in the Department of General and Transplant Surgery, T. Orłowski Institute, were enrolled in the study $(n=138)$. Deceased donor grafts were typed for HLA A, B, and DR. Kidney transplant recipients 
were typed for KIR and HLA A, B, C, and DR. Afterward, close monitoring of the occurrence of CMV infection, leucopenia, lymphocytopenia, and acute rejection episodes was performed for 720 days posttransplantation according to following scheme: every seven days in the first month, every two weeks in months 2 and 3, every four weeks in months 4, 5, and 6, and every three months later on. Additionally, CMV DNA-emia and kidney protocol biopsies were performed at the end of the third posttransplant month. Each case the clinical suspicion of CMV infection was verified by plasma CMV DNAemia. Recipients suspected of graft rejection were subjected to kidney biopsy. The eGFR was calculated by the abbreviated Modification of Diet in Renal Disease equation. A total of $61.6 \%$ of the recipients received induction therapy, and $87.7 \%$ received triple maintenance therapy with a combination of prednisone, tacrolimus, and antimetabolite (mycophenolatemofetil or mycophenolate sodium; Table 1). According to the institutional protocol, all of the patients received antiviral prophylaxis. A total of $83 \%$ of the recipients received valganciclovir, with the dose adjusted to kidney graft function, and $17 \%$ were given acyclovir (600 mg/day) for at least 90 days postoperatively. Based on the criteria that were recommended by the American Society of Transplantation for use in clinical trials, the following definitions applied:CMV infection (CMV DNAemia regardless of symptoms) and CMV disease (evidence of CMV infection with attributable symptoms) [36]. All rejection episodes were proven by biopsy, with the diagnosis based on Banff 2017criteria [37]. T-cell-mediated rejection was initially treated with $500 \mathrm{mg}$ methyloprednisolone intravenously for three consecutive days; if resistance to this treatment was evident, then the patients were treated with thymoglobulin. Antibody-mediated rejection episodes were treated with intravenous methyloprednisolone, immunoglobulin, and/or plasmapheresis.

The study was approved by the Ethical Committee of the Medical University of Warsaw (KB/114/2014) and complied with the provisions of Good Clinical Practice Guidelines and the Declaration of Helsinki. All of the patients provided informed consent prior to participation in the study.

\subsection{Procedures}

\subsubsection{KIR and HLA Genotyping}

Donors and recipients were typed for HLA-A, HLA-B, and HLA-DR-B1 by sequence-specific primer polymerase chain reaction (PCR). Peripheral blood specimens were collected, and mononuclear cell samples were isolated. DNA was extracted and stored at $-80 \mathrm{C}$ until use. DNA concentrations of each sample were determined using a UV-VIS NanoDrop Spectrophotometer (ThermoFisher Scientific, Wilmington, DE, USA). All further genotyping was performed retrospectively (i.e., at the end of follow up). DNA from mononuclear cells was used for KIR genotyping by applying a reverse sequence specific oligonucleotide (SSO) method according to the manufacturer's instructions (Lifecodes KIR Genotyping, Immucor Transplant Diagnostic, Stamford, CT, USA). The HLA-C and KIR genes were typed using Lifecodes HLA-SSO and KIR-SSO typing kits, respectively (ImmucorDgn, Lifecodes, Stamford, CT, USA) based on xMAP Luminex technology (Luminex, Austin, TX, USA) according to the manufacturer's instructions. The presence or absence of the following 16 KIR genes was identified: KIR2DL1, KIR2DL2, KIR2DL3, KIR2DL4, KIR2DL5, KIR2DS1, KIR2DS2, KIR2DS3, KIR2DS4, KIR2DS5, KIR3DL1, KIR3DL2, KIR3DL3, KIR3DS1, KIR2DP1, and KIR3DP1. KIR gene profiles were determined by the presence or absence of each KIR gene in a given individual. This method of KIR typing does not allow the direct determination of KIR2DL2 copy number. Instead, we used the allelic nature of KIR2DL2 and KIR2DL3 at the 2DL2/2DL3 locus to infer the number of copies of KIR2DL2. KIR2DS4 was typed for encoded cell-surface receptor (full) or a truncated protein variant with loss of the transmembrane and cytoplasmic domains (del) by GeneScan analysis with a 6-FAM labeled primer. The deleted variant of KIR2DS4 was not anchored to the cell membrane but was encoded for a soluble form of the protein that is potentially secreted and likely lacks function(s). We used the MATCH IT DNA program for analysis. 
The presence of KIR ligands in recipients was assessed by grouping each patient's HLA class I antigens according to defined specificities: patients were considered to have $\mathrm{C} 1$ group ligands if they possessed an HLA-C molecule with an asparagine at position 80 (e.g., HLA-Cw1, -Cw3, -Cw7, and $-\mathrm{Cw} 8), \mathrm{C} 2$ group ligands if they possessed an HLA-C molecule with a lysine residue at position 80 (e.g., HLA-Cw2,-Cw4,-Cw5, and -Cw6), and Bw4 ligands if their HLA-B antigens included at least one antigen with Bw4 specificity (e.g., HLA-B5, -B13, -B17, and -B27) as described elsewhere [13,38,39]. We defined KIR-A and KIR-B haplotypes as previously described. In brief, KIR-A haplotypes only contain KIR2DL1, KIR3DL1, and KIR2DS4 and four framework genes (KIR2DL4, KIR3DL2, KIR3DL3, and KIR2DL3). KIR-B haplotypes contain one or more of the following genes: KIR2DL2, KIR2DL5, KIR2DS1, KIR2DS2, KIR2DS3, KIR2DS5, and KIR3DS1.

\subsubsection{DNAemia}

Cytomegalovirus DNAemia was evaluated in plasma using a commercial quantitative nucleic acid amplification test (SmartCycler II, Cepheid AB, Sunnyvale, CA, USA) that from 2013 was calibrated to the 1st World Health Organization International Standard. The limit of detection was 50 copies $/ \mathrm{mL}$, with a linearity of 500-107 copies/mL.

\subsubsection{Detection of CMV-Specific Antibodies}

Specific serum anti-CMV IgG titers were measured using the ARCHITECT CMV IgG assay (Abbott Laboratories, Dublin, Ireland) according to the manufacturer's instructions. The ARCHITECT CMV IgG assay is a chemiluminescent microparticle immunoassay that is designed to have a precision of $\leq 10 \%$ total (total is the accumulation of within run, between run, and between day) coefficient of variation (CV) for representative specimens within the ranges of 6-60 AU/mL and 200-250 AU $/ \mathrm{mL}$. Among transplant recipients, relative sensitivity is $100 \%$ (lower $95 \%$ confidence limit of $91.96 \%$ ), and specificity is $100 \%$ (lower $95 \%$ confidence limit of $93.62 \%$; from the manufacturer's information brochure).

\subsection{Statistical Analyses}

Qualitative variables were compared using the $\chi^{2}$ test and Fisher's exact test, respectively, to the sample size. To measure the fraction of events, the Kaplan-Meier model was used. This model estimates and tests survival over time under different factors. Quantitative variables were summarized by medians (ranges) because the parameters did not follow a normal distribution, and they were compared using the Wilcoxon Rank-Sum test. Multidimensional analysis was performed with the generalized logistic regression model (GLM). To assess the goodness-of-fit and select the optimal model, the Akaike Information Criterion statistic was used. Values of $p<0.05$ were considered statistically significant. The data were analyzed using SAS/STAT 14.3 [SAS Institute Inc., Cary, NC, USA] software.

\section{Conclusions}

In summary, our results suggest that genotyping recipients for the KIR2DS2, KIR2DL2, and KIR2DL3 genes may help predict the occurrence of posttransplant CMV infection. Other factors, such as graft function, time of antiviral prophylaxis initiation, lymphocyte blood count, and pretransplant serostatus, were independent predictive factors for the occurrence of CMV. Currently, the risk stratification for posttransplant CMV infection is primarily based on the assessment of D/R CMV serostatus prior to transplantation. In our opinion, this strategy could be improved by implementing other biomarkers that are predictive of CMV infection after kidney transplantation, which would allow for more personalized management. The assessment of KIR2DS2 prior to transplantation could potentially be used in combination with $\mathrm{D} / \mathrm{R}$ serostatus to more accurately predict the risk of $\mathrm{CMV}$ infection, including the precise identification of transplant individuals who require a longer duration of antiviral prophylaxis therapy in individuals who do not have KIR2DS2. In conclusion, our findings confirm that KIR/HLA genotypes play a significant role in anti-CMV immunity and suggest the 
contribution of not only environmental but also genetic factors in the incidence of CMV infection after kidney transplantation.

Author Contributions: D.D.-M. contributed to the research design, performance of the research, analysis, interpretation of data, drafting the work, writing of the paper. A.P.-P. contributed to the research design, revising the work critically, writing of the paper. J.G., M.C. and A.O. contributed to the performance of the research, reviewed all the medical records and collected CMV data. A.S.-J. contributed to the and KIR, HLA genotyping and evaluated CMV data to obtain the diagnosis of CMV infection. A.O., J.P., M.C., J.G. collected completing data and blood samples and assisted in collection of the other clinical data. M.D., J.N. and M.K. reviewed and made improvements in the manuscript. The final manuscript was read and approved by all authors.

Funding: The study was financially supported by statutory funds from the Medical University of Warsaw. DDM was supported by a Roche research grant. The funders had no role in study design, data collection and analysis, decision to publish, or preparation of the article.

Conflicts of Interest: The authors declare that they have no financial or non-financial competing interests. The sponsors had no role in the design, execution, interpretation, or writing of the study.

\section{Abbreviations}

ABMR Antibody-mediated rejection

AU Arbitrary unit

CI Confidence interval

CMV Cytomegalovirus

D Donor

eGFR Estimated glomerular filtration rate

GLM Generalized linear models

HLA Human leukocyte antigen

KIR Killer-cell immunoglobulin-like receptor

NK Natural killer

OR Odds ratio

PCR Polymerase chain reaction

R Recipient

SAS Statistical analysis system

SD Standard deviation

SSO Sequence specific olinucleotides

TCMR T cell-mediated rejection

\section{References}

1. Razonable, R.R.; Humar, A. Cytomegalovirus in solid organ transplantation. Am. J. Transplant. 2013, 13 (Suppl. 4), 93-106. [CrossRef] [PubMed]

2. Razonable, R.R.; Rivero, A.; Rodriguez, A.; Wilson, J.; Daniels, J.; Jenkins, G.; Larson, T.; Hellinger, W.C.; Spivey, J.R.; Paya, C.V. Allograft rejection predicts the occurrence of late-onset cytomegalovirus (CMV) disease among CMV-mismatched solid organ transplant patients receiving prophylaxis with oral ganciclovir. J. Infect. Dis. 2001, 184, 1461-1464. [CrossRef] [PubMed]

3. Humar, A.; Lebranchu, Y.; Vincenti, F.; Blumberg, E.A.; Punch, J.D.; Limaye, A.P.; Abramowicz, D.; Jardine, A.G.; Voulgari, A.T.; Ives, J.; et al. The efficacy and safety of 200 days valganciclovir cytomegalovirus prophylaxis in high-risk kidney transplant recipients. Am. J. Transplant. 2010, 10, 1228-1237. [CrossRef] [PubMed]

4. Schoeppler, K.E.; Lyu, D.M.; Grazia, T.J.; Crossno, J.T., Jr.; Vandervest, K.M.; Zamora, M.R. Late-onset cytomegalovirus (CMV) in lung transplant recipients: Can CMV serostatus guide the duration of prophylaxis? Am. J. Transplant. 2013, 13, 376-382. [CrossRef]

5. Santos, C.A.; Brennan, D.C.; Fraser, V.J.; Olsen, M.A. Delayed-onset cytomegalovirus disease coded during hospital readmission after kidney transplantation. Transplantation 2014, 98, 187-194. [CrossRef]

6. Venema, H.; van den Berg, A.P.; van Zanten, C.; van Son, W.J.; van der Giessen, M.; The, T.H. Natural killer cell responses in renal transplant patients with cytomegalovirus infection. J. Med. Virol. 1994, 42, 188-192. [CrossRef] [PubMed] 
7. Wilson, M.J.; Torkar, M.; Haude, A.; Milne, S.; Jones, T.; Sheer, D.; Beck, S.; Trowsdale, J. Plasticity in the organization and sequences of human KIR/ILT gene families. Proc. Natl. Acad. Sci. USA 2000, 97, 4778-4783. [CrossRef]

8. Marsh, S.G.; Parham, P.; Dupont, B.; Geraghty, D.E.; Trowsdale, J.; Middleton, D.; Vilches, C.; Carrington, M.; Witt, C.; Guethlein, L.A.; et al. Killer-cell immunoglobulin-like receptor (KIR) nomenclature report, 2002. Tissue Antigens 2003, 62, 79-86. [CrossRef]

9. Lanier, L.L. NK cell recognition. Annu. Rev. Immunol. 2005, 23, 225-274. [CrossRef]

10. Hsu, K.C.; Liu, X.R.; Selvakumar, A.; Mickelson, E.; O’Reilly, R.J.; Dupont, B. Killer Ig-like receptor haplotype analysis by gene content: Evidence for genomic diversity with a minimum of six basic framework haplotypes, each with multiple subsets. J. Immunol. 2002, 169, 5118-5129. [CrossRef]

11. Hadaya, K.; de Rham, C.; Bandelier, C.; Bandelier, C.; Ferrari-Lacraz, S.; Jendly, S.; Berney, T.; Buhler, L.; Kaiser, L.; Seebach, J.D.; et al. Natural killer cell receptor repertoire and their ligands, and the risk of CMV infection after kidney transplantation. Am. J. Transplant. 2008, 8, 2674-2683. [CrossRef] [PubMed]

12. Stern, M.; Elsässer, H.; Hönger, G.; Steiger, J.; Schaub, S.; Hess, C. The number of activating KIR genes inversely correlates with the rate of CMV infection/reactivation in kidney transplant recipients. Am. J. Transplant. 2008, 8, 1312-1317. [CrossRef] [PubMed]

13. Stern, M.; Hadaya, K.; Hönger, G.; Martin, P.Y.; Steiger, J.; Hess, C.; Villard, J.; Stern, M.; Hadaya, K.; Hönger, G.; et al. Telomeric rather than centromeric activating KIR genes protect from cytomegalovirus infection after kidney transplantation. Am. J. Transplant. 2011, 11, 1302-1307. [CrossRef] [PubMed]

14. Zaia, J.A.; Sun, J.Y.; Gallez-Hawkins, G.M.; Thao, L.; Oki, A.; Lacey, S.F.; Dagis, A.; Palmer, J.; Diamond, D.J.; Forman, S.J.; et al. The effect of single and combined activating killer immunoglobulin-like receptor genotypes on cytomegalovirus infection and immunity after hematopoietic cell transplantation. Biol. Blood Marrow Transplant. 2009, 15, 315-325. [CrossRef] [PubMed]

15. Gallez-Hawkins, G.M.; Franck, A.E.; Li, X.; Thao, L.; Oki, A.; Gendzekhadze, K.; Dagis, A.; Palmer, J.; Nakamura, R.; Forman, S.J.; et al. Expression of activating KIR2DS2 and KIR2DS4 genes after hematopoietic cell transplantation: Relevance to cytomegalovirus infection. Biol. Blood Marrow Transplant. 2011, 17, 1662-1672. [CrossRef] [PubMed]

16. Van Duin, D.; Avery, R.K.; Hemachandra, S.; Yen-Lieberman, B.; Zhang, A.; Jain, A.; Butler, R.S.; Barnard, J.; Schold, J.D.; Fung, J.; et al. KIR and HLA Interactions Are Associated with Control of Primary CMV Infection in SolidOrgan Transplant Recipients. Am. J. Transplant. 2014, 14, 156-162. [CrossRef] [PubMed]

17. Di Bona, D.; Accardi, G.; Aiello, A.; Bilancia, M.; Candore, G.; Colomba, C.; Caruso, C.; Duro, G.; Gambino, C.M.; Macchia, L.; et al. Association between $\gamma$ marker, human leucocyte antigens and killerimmunoglobulin-likereceptors and the naturalcourse of human cytomegalovirusinfection: A pilot study performed in a Sicilian population. Immunology 2018, 153, 523-531. [CrossRef]

18. Tuttolomondo, A.; Colomba, C.; Di Bona, D.; Casuccio, A.; Di Raimondo, D.; Clemente, G.; Arnao, V.; Pecoraro, R.; Ragonese, P.; Aiello, A.; et al. HLA and killer cell immunoglobulin-like receptor (KIRs) genotyping in patients with acute viral encephalitis. Oncotarget 2018, 9, 17523-17532. [CrossRef] [PubMed]

19. Askar, M.; Avery, R.; Corey, R.; Lopez, R.; Thomas, D.; Pidwell, D.; Eghtesad, B.; Miller, C.; Fung, J.; Zein, N.N. Lack of killerimmunoglobulin-like receptor 2DS2 (KIR2DS2) and KIR2DL2 is associated with poor responses to therapy of recurrent hepatitis C virus in liver transplant recipients. Liver Transpl. 2009, 15, 1557-1563. [CrossRef] [PubMed]

20. Naiyer, M.M.; Cassidy, S.A.; Magri, A.; Cowton, V.; Chen, K.; Mansour, S.; Kranidioti, H.; Mbirbindi, B.; Rettman, P.; Harris, S.; et al. KIR2DS2recognizes conserved peptidesderived from viral helicases in the context of HLA-C. Sci. Immunol. 2017, 2, eaal5296. [CrossRef] [PubMed]

21. Winter, C.C.; Gumperz, J.E.; Parham, P.; Long, E.O.; Wagtmann, N. Direct Binding and Functional Transfer of NK Cell Inhibitory Receptors Reveal Novel Patterns of HLA-C Allotype Recognition. J. Immunol. 1998, 161, 571-577. [PubMed]

22. Saito, H.; Umemura, T.; Joshita, S.; Yamazaki, T.; Fujimori, N.; Kimura, T.; Komatsu, M.; Matsumoto, A.; Tanaka, E.; Ota, M. KIR2DL2 combined with HLA-C1 confers risk of hepatitis C virus-related hepatocellular carcinoma in younger patients. Oncotarget 2018, 9, 19650-19661. [CrossRef] [PubMed]

23. Shan, Z.; Huang, J.; Liao, Q.; Huang, K.; Wang, M.; Xu, R.; Tang, X.; Zhang, W.; Nelson, K.; Fu, Y.; et al. Association of killer cell immunoglobulin like receptors with spontaneous clearance of hepatitisC virus in the Chinese population. Transfusion 2018, 58, 1028-1035. [CrossRef] [PubMed] 
24. Bao, X.; Hanson, A.L.; Madeleine, M.M.; Wang, S.S.; Schwartz, S.M.; Newell, F.; Pettersson-Kymmer, U.; Hemminki, K.; Tiews, S.; Steinberg, W.; et al. HLA and KIR Associations of Cervical Neoplasia. J. Infect. Dis. 2018, 218, 2006-2015. [CrossRef] [PubMed]

25. Saulquin, X.; Gastinel, L.N.; Vivier, E. Crystal structure of the human natural killer cell activating receptor KIR2DS2 (CD158j). J. Exp. Med. 2003, 197, 933-938. [CrossRef] [PubMed]

26. Stewart, C.A.; Laugier-Anfossi, F.; Vély, F.; Saulquin, X.; Riedmuller, J.; Tisserant, A.; Gauthier, L.; Romagné, F.; Ferracci, G.; Arosa, F.A.; et al. Recognition of peptide MHC class I complexes by activating killer immunoglobulin-like receptors. Proc. Natl. Acad. Sci. USA 2005, 102, 13224-13229. [CrossRef] [PubMed]

27. Michelo, C.M.; van der Meer, A.; Tijssen, H.J.; Zomer, R.; Stelma, F.; Hilbrands, L.B.; Joosten, I. KIR and Human Leukocyte Antigen Genotype Associated Risk of Cytomegalovirus Disease in Renal Transplant Patients. Transplantation 2015, 99, 1506-1513. [CrossRef] [PubMed]

28. Gonzalez, A.; Schmitter, K.; Hirsch, H.H.; Garzoni, C.; van Delden, C.; Boggian, K.; Mueller, N.J.; Berger, C.; Villard, J.; Manuel, O.; et al. Swiss Transplant Cohort Study. KIR-associated protection from CMV replication requires pre-existing immunity: A prospective study in solid organ transplant recipients. Genes Immun. 2014, 15, 495-499. [CrossRef]

29. Cook, M.; Briggs, D.; Craddock, C.; Mahendra, P.; Milligan, D.; Fegan, C.; Darbyshire, P.; Lawson, S.; Boxall, E.; Moss, P. Donor KIR genotype has a major influence on the rate of cytomegalovirus reactivation following T-cell replete stem cell transplantation. Blood 2006, 107, 1230-1232. [CrossRef]

30. Helantera, I.; Lautenschlager, I.; Koskinen, P. The risk of cytomegalovirus recurrence after kidney transplantation. Transpl. Int. 2011, 24, 1170-1178. [CrossRef]

31. Kato, S.; Chmielewski, M.; Honda, H.; Pecoits-Filho, R.; Matsuo, S.; Yuzawa, Y.; Tranaeus, A.; Stenvinkel, P.; Lindholm, B. Aspect of Immune Dysfunction in End -stage Renal Disease. CJASN 2008, 3, 1526-1533. [CrossRef] [PubMed]

32. Deborska-Materkowska, D.; Perkowska-Ptasinska, A.; Sadowska, A.; Gozdowska, J.; Ciszek, M.; Serwanska-Swietek, M.; Domagala, P.; Miszewska-Szyszkowska, D.; Sitarek, E.; Jozwik, A.; et al. Diagnostic utility of monitoring cytomegalovirus-specific immunity by QuantiFERON-cytomegalovirus assay in kidney transplant recipients. BMC Infect. Dis. 2018, 18, 179. [CrossRef] [PubMed]

33. Fernández-Ruiz, M.; López-Medrano, F.; Romo, E.M.; Allende, L.M.; Meneu, J.C.; Fundora Suarez, Y.; San-Juan, R.; Lizasoain, M.; Paz-Artal, E.; Aguado, J.M. Pretransplant lymphocyte count predicts the incidence of infection during the first two years after liver transplantation. Liver Transpl. 2009, 15, 1209-1216. [CrossRef] [PubMed]

34. Nierenberg, N.E.; Poutsiaka, D.D.; Chow, J.K.; Cooper, J.; Price, L.L.; Freeman, R.B.; Rohrer, R.; Snydman, D.R. Pretransplant lymphopenia is novel prognostic factor in cytomegalovirus and non-0cytomegalovirus invasive infections after liver transplantation. Liver Transpl. 2014, 20, 1497-1507. [PubMed]

35. San-Juan, R.; Navarro, D.; García-Reyne, A.; Montejo, M.; Muñoz, P.; Carratala, J.; Len, O.; Fortun, J.; Muñoz-Cobo, B.; Gimenez, E.; et al. Effect of delaying prophylaxis against CMV in D+/R- solid organ transplant recipients in the development of CMV-specific cellular immunity and occurrence of late CMV disease. J. Infect. 2015, 71, 561-570. [CrossRef] [PubMed]

36. Kotton, C.N.; Kumar, D.; Caliendo, A.M.; Huprikar, S.; Chou, S.; Danziger-Isakov, L.; Humar, A.; The Transplantation Society International CMV Consensus Group. The third international consensus guidelines on the management of cytomegalovirus in solid-organ transplantation. Transplantation 2018, 102, 900-931. [CrossRef]

37. Roufosse, C.; Simmonds, N.; Clahsen-van Groningen, M.; Haas, M.; Henriksen, K.J.; Horsfield, C.; Loupy, A.; Mengel, M.; Perkowska-Ptasińska, A.; Rabant, M.; et al. A 2018 Reference Guide to the Banff Classification of Renal Allograft Pathology. Transplantation 2018, 102, 1795-1814. [CrossRef]

38. Hsu, K.C.; Chida, S.; Geraghty, D.E.; Dupont, B. The killer cell immunoglobulin-like receptor (KIR) genomic region: Gene-order, haplotypes and allelic polymorphism. Immunol. Rev. 2002, 190, 40-52. [CrossRef]

39. Uhrberg, M.; Valiante, N.M.; Shum, B.P.; Shilling, H.G.; Lienert-Weidenbach, K.; Corliss, B.; Tyan, D.; Lanier, L.L.; Parham, P. Human diversity in killer cell inhibitory receptor genes. Immunity 1997, 7, 753-763. [CrossRef]

(C) 2019 by the authors. Licensee MDPI, Basel, Switzerland. This article is an open access article distributed under the terms and conditions of the Creative Commons Attribution (CC BY) license (http://creativecommons.org/licenses/by/4.0/). 